\title{
Deterministic Propagation Approach for Millimeter Wave Outdoor Smart Parking Solution Deployment ${ }^{\dagger}$
}

\author{
Fidel Alejandro Rodríguez-Corbo ${ }^{1}$, Leyre Azpilicueta ${ }^{1, *}{ }^{\text {, }}$ Mikel Celaya-Echarri ${ }^{1}$, Peio Lopez- \\ Iturri ${ }^{2,3}$, Ana V. Alejos ${ }^{4}$ and Francisco Falcone ${ }^{2,3}$ \\ 1 School of Engineering and Sciences, Tecnologico de Monterrey, 64849 Monterrey, Mexico; \\ alek.bsb@gmail.com (F.A.R.-C.); mikelcelaya@gmail.com (M.C.-E.) \\ 2 Department of Electric, Electronic and Communication Engineering, Public University of Navarre, 31006 \\ Pamplona, Spain; peio.lopez@unavarra.es (P.L.-I.); francisco.falcone@unavarra.es (F.F.) \\ 3 Institute of Smart Cities, Public University of Navarre, 31006 Pamplona, Spain \\ 4 Department of Teoría de la Señal y Comunicación, University of Vigo, 36310 Vigo, Spain, \\ analejos@uvigo.es \\ * Correspondence: leyre.azpilicueta@tec.mx; Tel.: +52-81582082; Fax: +52-8183597211 \\ + Presented at the 7th Electronic Conference on Sensors and Applications, 15-30 November 2020; Available \\ online: https://ecsa-7.sciforum.net/.
}

Published: 15 November 2020

\begin{abstract}
Impact factor as efficiency or sustainability are entirely correlated to the continuous development of the smart city concept technology application. Intelligent transportation systems (ITS) and particularly autonomous vehicles are expected to play an important role in this challenging environment. Fast and secure connections will be pivotal in order to achieve this new vehicular communications' application era. The use of millimeter-wave (mmWave) frequency range is the most promising approach to allow these real-time high-demand applications which requires higher bandwidth with the minimum possible latency. However, an in-depth mmWave's channel characterization of the environment is required for a proper mmWave based solution deployment. In this work, a complete radio wave propagation channel characterization for a mmWave smart parking solution deployment in a complex outdoor environment has been assessed, at $28 \mathrm{GHz}$ frequency band. The considered scenario is a parking lot placed in an open free university campus area surrounded by inhomogeneous vegetation. Vehicle and vegetation density within the scenario, in terms of inherent transceivers density and communication impairments, leads to overall system operation challenges, given by multiple communication links operation at line-of-sight (LOS) and non-line-of-sight (NLOS) conditions. By means of an in-house developed 3D ray launching (3D-RL) algorithm, the impact of variable vegetation density is addressed, providing precise modelling estimations of large-scale multipath propagation effects in terms of received power levels and path loss. The obtained results along with the proposed simulation methodology, can aid in an adequate characterization of the mmWave communication channel for new vehicular communications networks, applications and deployments, considering outdoor conditions as well as the impact of different vegetation densities, for current as well as for future wireless technologies.
\end{abstract}

Keywords: propagation modelling; millimeter wave; 3D Ray Launching; smart parking; vegetation environment; vehicular communications

\section{Introduction}

With the hope to guide our future towards greater sustainability, the use of the recent advances in communication technologies will empower the dream of real smart cities. In this envisioned environment, technology and creativity come together with the aim of making society more efficient and sustainable. As part of this goal, intelligent transportation systems (ITS) will employ the 
application of sensing, analysis, and control over the vehicular environment entities (e.g., vehicles, traffic signs, parking lots, route controllers) to make transportation more efficient and secure. Considering that by 2050 about $68 \%$ of the world's population will live in cities [1], it is not surprising that ITS will be essential within the main structure of a smart city. With the advent of autonomous vehicles within this ITS context, the tasks of driving and operating the vehicle will be less and less in the hands of the human driver. At that point, fully automated vehicles will communicate wirelessly to other vehicles (V2V) or infrastructures (V2I) as a way to gather the required information to act under different conditions, environments and constraints. Although there are already so-called smart parking lots, it is with the arrival of fully autonomous vehicles that these spaces will achieve their full potential. The requirements of autonomous vehicles in an intelligent parking environment are closely related to the demands of automated driving on a daily basis: utilities such as multiple sensors and raw cameras data and processes interacting between V2V and V2I communication links.

In this sense, the demands for higher capacity/bandwidth and low latencies in communication systems increase dramatically each year, where predictions for future smart cities place more stress in these aforementioned demands. In the autonomous vehicular environment domain, the need for data access in real-time, raw video feed information, real-time route control, as well as high throughput and time constraints, has led to the proposal of millimeter-wave (mmWave) frequency bands as a way to tackle these requirements. The frequency spectrum in mmWave bands provide large bandwidths that can allocate communications systems achieving high throughput and low latencies; the adoption of these bands is envisioned to be a fundamental part of the 5 th generation (5G) of future communications. Despite these advantages, communication in the mmWave frequency bands presents high penetration losses and poor diffraction, which imposes many challenges for its implementation. Propagation characteristics in $\mathrm{mmWave}$ bands are significantly different from those in micrometric frequencies; therefore, these events have led to an increase in both academic and industry interest in these frequencies bands. Thus, it is undoubtedly essential to characterize communication in these high frequency bands, specially under the effects of non-line of sight (NLOS). It must be remarked that the shadowing of the radio signal in the mmWave range can induce high losses in the received signal strength, more aggravated with the use of directional beams as it is expected in the mmWave Multiple Input Multiple Output (MIMO) communication systems.

Particularly in the autonomous vehicular context, the study of blockage in mmWave frequency bands is mostly encompassing blocking by other vehicles or buildings, and in some cases taking into account the vegetation. In addition to this, most investigations address typical environments like rural, urban, and highways; but works focused on other less common circumstances such as parking lots, tunnels, and roundabout are scarce. Sceneries such as smart parking spaces have been addressed in the literature at micrometric frequencies in works such as [2,3]; in these papers, an empirical approach has been followed, showing the obtained measurement results in two indoor parking areas. Regarding the mmWave frequency range, the work presented by [4] analyzes the path loss (PL) in an indoor parking environment at frequencies of $26.5 \mathrm{GHz}$ and $38.5 \mathrm{GHz}$. In the literature, outdoor parking spaces are less considered, usually characterized by spacious zones and often with abundant inhomogeneous vegetation, challenging in terms of channel modelling and propagation characterization. In [5], the path losses in an outdoor parking environment at $28 \mathrm{GHz}$ and $38 \mathrm{GHz}$ is inspected employing a measurement campaign and the PL adjustment to the close-in models (CI) free space reference and the floating-intercept (FI) PL model is provided. Also, due to the presence of vegetation in outdoor parking areas, the communication channel is affected by an increase in the probabilities of NLOS, temporal, and angular dispersion parameters [6]. In general, the impact of vegetation density in the mmWave frequency range is still an issue that needs further investigation; the vegetation density effects over the wireless channel characteristics is an important aspect to take into account in this technology requirements as a communication medium in outdoor environments.

In this work, the mmWave frequency band vegetation density impact in an outdoor parking lot is assessed by means of an in-house developed 3D Ray Launching (3D-RL) algorithm. Discussion of the influence of vegetation density is presented, showing that the obtained results can aid in an 
adequate characterization of the mmWave communication channel for new vehicular communications networks, applications and deployments, considering outdoor conditions.

\section{Scenario Description}

As commonly found in outdoor parking lots, trees planted in dividing parking lots serve both as decorative and to provide shade. These trees' barriers are frequently composed of a single line of trees and shrubs. The location and densities of these obstacles can vary immensely, and in different distributions. In this work, in order to analyze these channel modeling impairments and effects, an outdoor parking lot placed inside the Monterrey campus of Tecnologico de Monterrey, has been considered. The represented vegetation is composed of $6.2 \mathrm{~m}$ height trees with vast foliage and small $1.2 \mathrm{~m}$ bushes. For comparison, two different scenarios are presented, with differing volumetric vegetation densities. In the first one, trees and shrubs are distributed continuously and occupying the entire available area of the barrier between the parking lot. This distribution density constitutes a total of 8 trees and 9 shrubs; covering a volumetric density of $378.8 \mathrm{~m}^{3}$. For the second scenario, the distribution density is decreased to approximately one third (33\%), composed of 3 trees and 3 shrubs, for an approximate volumetric density of $138.4 \mathrm{~m}^{3}$. As an example, Figure 1 presents the rendered view of the high vegetation density considered scenario in the parking lot.

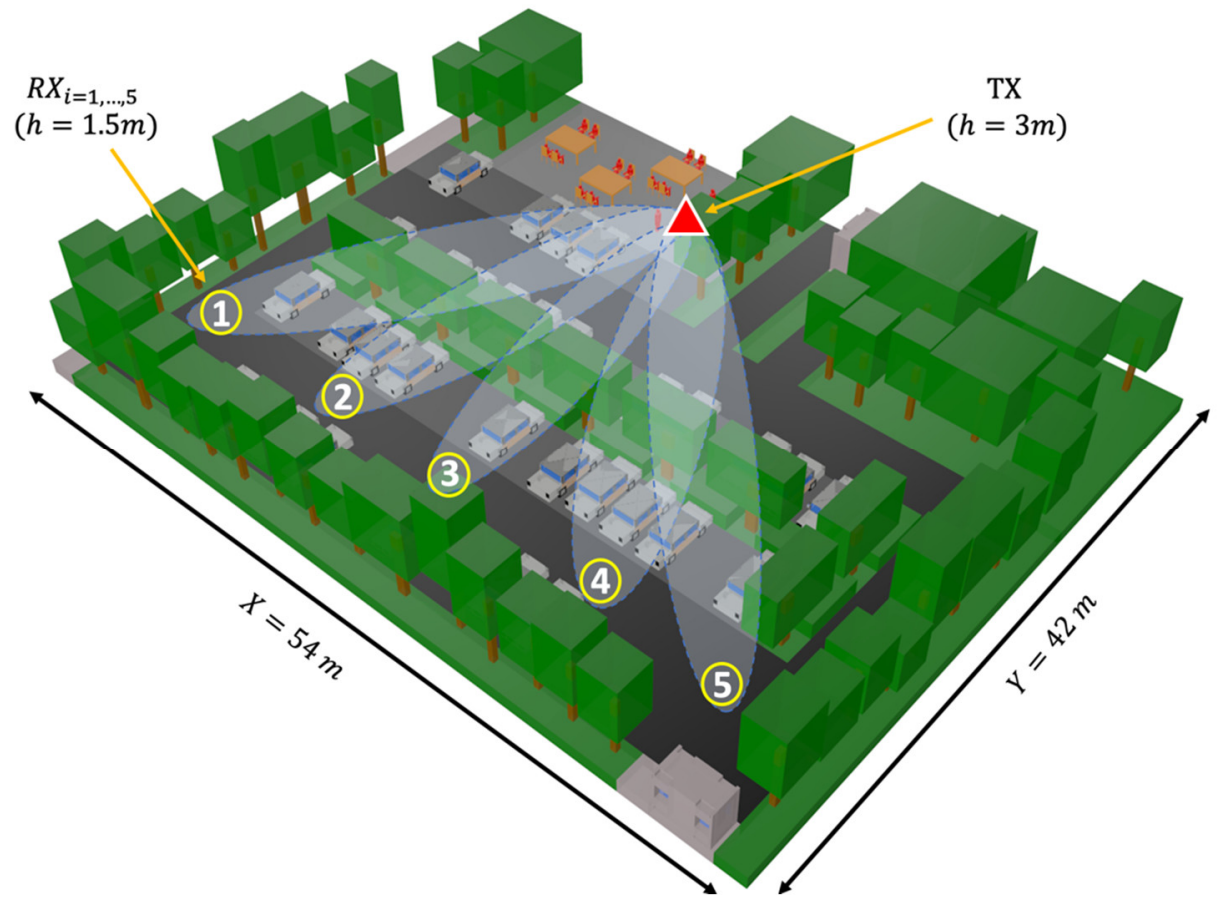

Figure 1. Rendered view of the parking lot considered scenario for simulation. Transmitter and receiver positions are presented, as well as the different communications beams assessed.

An in-house deterministic 3D-RL algorithm has been used to assess the vegetation density impact at $28 \mathrm{GHz}$ frequency band for the smart parking solution deployment. The detailed information of the 3D-RL algorithm and its validation in vehicular environments can be found in $[7,8]$. For the simulation purpose, a transmitter antenna placed in a streetlight at $3 \mathrm{~m}$ height has been considered, as well as several NLOS challenging receiver locations in the scenario. Different directional beams have been assessed, aiming at points located on a typical vehicular receiver $(1.5 \mathrm{~m}$ height) on the other side of the vegetation barrier according to the arrangement presented in Figure 1. The most relevant simulation parameters are presented in Table 1. 
Table 1. Simulation parameters.

\begin{tabular}{cc}
\hline Parameters & Values \\
\hline TX Power & $25 \mathrm{dBm}$ \\
Carrier frequency & $28 \mathrm{GHz}$ \\
Bit Rate & $4.62 \mathrm{Gbps}$ \\
Antenna type/Gain & Directional $/ 20 \mathrm{~dB}$ \\
3D Ray Launching: angular resolution/Rebounds & $0.2 \mathrm{degree} / 6$ \\
Scenario size/Unitary volume analysis & $(54 \times 42 \times 15) \mathrm{m} / 1 \mathrm{~m}^{3}(1 \times 1 \times 1) \mathrm{m}$ \\
\hline
\end{tabular}

\section{Results and Discussions}

As shown in Figure 1, the radiation beams are launched to locations placed beyond the tree barrier, taking into account the range of paths in each beam, the worst-case scenario is chosen as a reference for path losses through the vegetation. In the shortest direction, the tree barrier is located at a distance of approximately $11 \mathrm{~m}$ from the transmitter. Using this metric, the path losses are obtained in both scenarios (high vegetation density and low vegetation density) in each beam. Figure 2 show both scenarios in terms of the PL across each of the beams. In addition, the fit to the Close-In path loss model for each radiation pattern is plotted, according to Equation (1).

$$
P L_{C I}(f, d)[d B]=F S L P(f, d(1 m))[d B]+10 n \log _{10}(d)+X_{\sigma},
$$

where $F S L P(f, d(1 \mathrm{~m}))=20 \log _{10} \frac{4 \pi f}{c}$ denotes the free-space path loss in $\mathrm{dB}$ at a TransmitterReceiver (TR) separation distance of $1 \mathrm{~m}$ at the carrier frequency $\mathrm{f}$; $\mathrm{c}$ is the speed of light, $\mathrm{n}$ is the path loss exponent (PLE), $\mathrm{d}$ is the $3 \mathrm{D}$ separation distance between the transmitter and the receiver, and $X_{\sigma}$ is the shadow fading standard deviation for large-scale signal fluctuations. The PL described in Figure 2 shows attenuations under line-of-sight (LOS) conditions up to $11 \mathrm{~m}$, where the tree barrier is located. Under these conditions, the wireless communications channel behaves with a mean PL exponent $(\overline{P L E})$ between 1.7 and 1.8, describing conditions better than free space losses; this is related to strong reflections on the bodies of parked vehicles. Beyond the barrier, the wireless link is strongly affected in both coefficient parameters, PLE and path dispersion. In the case of the low vegetation density scenario (Figure 2a), the PLE attains an average of 2.5, and the standard deviation reaches an average value of $9.08 \mathrm{~dB}$. For the scenario with high vegetation density (Figure $2 b$ ), the average PLE approach a score of 2.79 , and the average standard deviation is around $11.5 \mathrm{~dB}$. This difference in PLE is represented in Figure 3, where the effects on PL in both scenarios are shown, starting from the $12 \mathrm{~m}$ mark (after the vegetation barrier), together with the PL fit to the CI model.

The difference in the average settings in the different scenarios is $4 \mathrm{~dB}$ taking into account the worst scenario in both situations (which in the case of low vegetation may also include obstructions). In the case of standard deviation, the high-density scheme incurs an increase of approximately $2.5 \mathrm{~dB}$ over the low-density scenario. Taking into account the continuous distribution of trees in the case of maximum volumetric density and the reduction to one-third of this density, Table 2 reflects the summary of the effects analyzed under these conditions.

Table 2. Simulation results for the differing vegetation volumetric densities.

\begin{tabular}{cccc}
\hline Parameter & High density & Low density & Difference \\
\hline$\overline{P L E}$ & 2.79 & 2.5 & 0.29 \\
$\overline{X_{\sigma}}$ & $11.56 \mathrm{~dB}$ & $9.08 \mathrm{~dB}$ & $2.48 \mathrm{~dB}$ \\
\hline
\end{tabular}




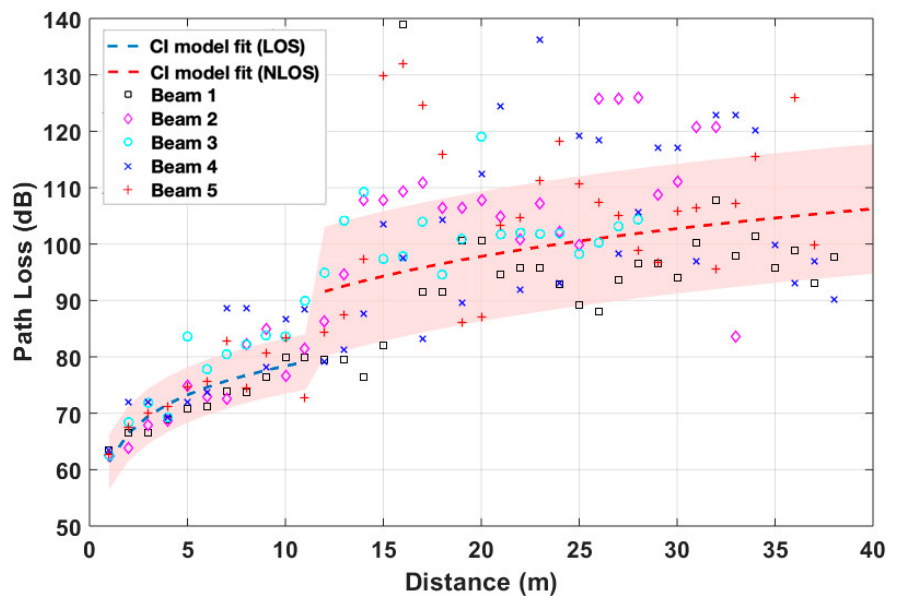

(a)

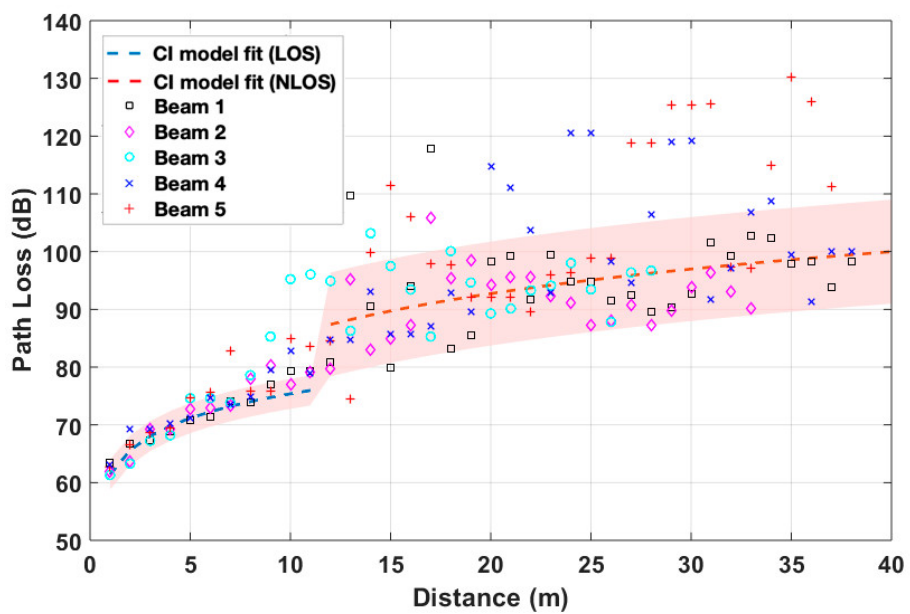

(b)

Figure 2. Path Loss for all five beams and both vegetation density, listed as: (a) High vegetation density scenario; (b) Low vegetation density scenario.

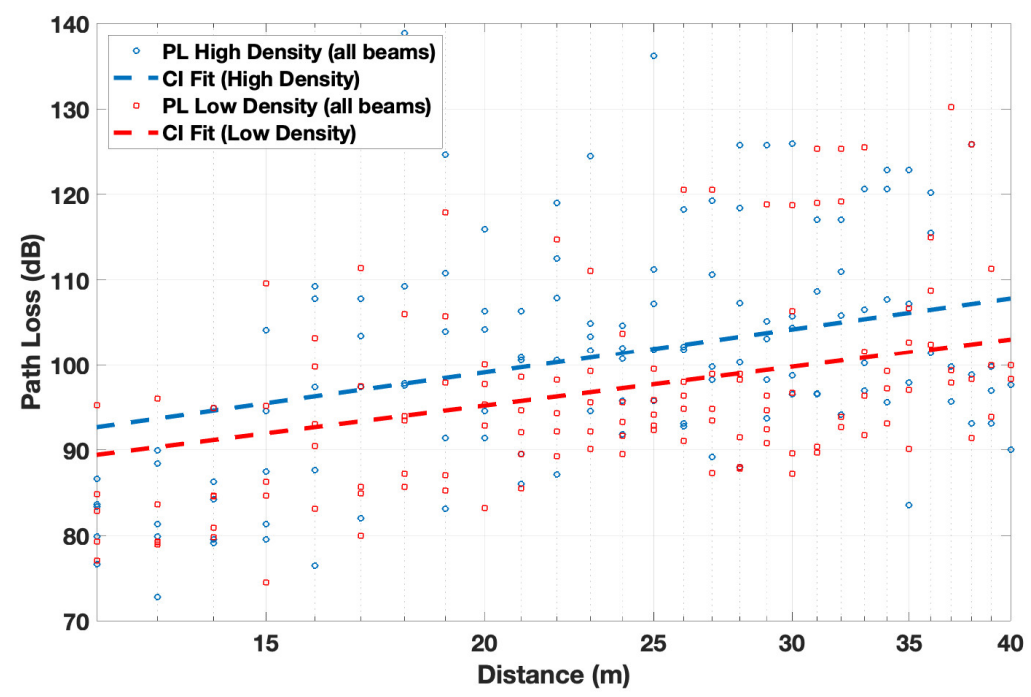

Figure 3. CI path loss model fit for both scenarios. 


\section{Conclusions}

In outdoor parking scenarios, the use of barriers with abundant vegetation is common practice. The communication of directional beams in the mmWave frequency bands for V2I communications in smart parking lots can be strongly affected by these vegetation barriers. The characterization of the different vegetation densities and their effects on the large-scale channel parameters can grant important information throughout the deployment of communications under outdoor conditions. This article presents the worst-case PL analysis considering different vegetation densities. From the results, the high vegetation density case presents an increase in the PL average of $4 \mathrm{~dB}$ and an increase in the standard deviation of $2.5 \mathrm{~dB}$ over the low vegetation density scenario. The obtained results can be applied in similar vegetation conditions to characterize and design V2I communication systems in smart parking lots under vegetation obstructions effects. As a future work, a further study is intended to expand this presented analysis to wider varieties of vegetation and vehicle densities, providing coverage/capacity analysis and precise modelling estimations of small-scale and large-scale multipath propagation effects in terms of received power levels and time domain characteristics.

Author Contributions: Conceptualization, L.A., M.C.-E. and F.A.R.-C.; methodology, L.A.; software, L.A., F.A.R.-C.; validation, M.C.-E. and F.A.R.-C.; formal analysis, P.L-I. and F.A.R.-C.; investigation, M.C.-E. and F.A.R.-C.; resources, L.A, A.V.A and F.F.; data curation, M.C.-E. and F.A.R.-C.; writing-original draft preparation, F.A.R.-C.; writing-review and editing, L.A. and M.C.-E.; visualization, M.C.-E. and P.L.-I.; supervision, L.A, A.V.A and F.F.; project administration, L.A.; funding acquisition, L.A, A.V.A and F.F. All authors have read and agreed to the published version of the manuscript.

Funding: Project RTI2018-095499-B-C31, funded by Ministerio de Ciencia, Innovación y Universidades, Gobierno de España (MCIU/AEI/FEDER, UE).

Acknowledgments: The authors would like to acknowledge the support and collaboration of the School of Engineering and Sciences at Tecnologico de Monterrey.

Conflicts of Interest: The authors declare no conflict of interest.

\section{References}

1. Nations, U.; Economic, D.o.; Affairs, S.; Division, P. World Urbanization Prospects The 2018 Revision; UN iLibrary: 2018.

2. Matolak, D.W.; Sun, R.; Liu, P. V2V channel characteristics and models for 5 GHz parking garage channels. In Proceedings of the 2015 9th European Conference on Antennas and Propagation, EuCAP 2015, Lisbon, Portugal, 13-17 April 2015; pp. 1-4.

3. Sun, R.; Matolak, D.W.; Liu, P. Parking garage channel characteristics at 5 GHZ for V2V applications. In Proceedings of the IEEE Vehicular Technology Conference, Las Vegas, NV, USA, 2-5 September 2013; pp. 1-5, doi:10.1109/VTCFall.2013.6692343.

4. Uyrus, A.; Turan, B.; Basar, E.; Coleri, S. Visible Light and mmWave Propagation Channel Comparison for Vehicular Communications. In Proceedings of the IEEE Vehicular Networking Conference, Los Angeles, CA, USA, 4-6 December 2019; doi:10.1109/VNC48660.2019.9062797.

5. Al-Samman, A.M.; Rahman, T.A.; Hindia, M.H.D.N.; Daho, A.; Hanafi, E. Path loss model for outdoor parking environments at $28 \mathrm{GHz}$ and $38 \mathrm{GHz}$ for $5 \mathrm{G}$ wireless networks. Symmetry 2018, 10, 672, doi:10.3390/sym10120672.

6. Zhang, P.; Yang, B.; Yi, C.; Wang, H.; You, X. Measurement-Based 5G Millimeter-Wave Propagation Characterization in Vegetated Suburban Macrocell Environments. IEEE Trans. Antennas Propag. 2020, 68, 5556-5567, doi: 10.1109/TAP.2020.2975365.

7. AAzpilicueta, L.; Rawat, M.; Rawat, K.; Ghannouchi, F.; Falcone, F. A Ray Launching-Neural Network Approach for Radio Wave Propagation Analysis in Complex Indoor Environments. IEEE Trans. Antennas Propag. 2014, 62, 2777-2786.

8. Azpilicueta, L.; Lopez-Iturri, P.; Zuñiga-Mejia, J.; Celaya-Echarri, M.; Rodríguez-Corbo, F.A.; VargasRosales, C.; Aguirre, E.; Michelson, D.G.; Falcone, F. Fifth-Generation (5G) mmWave Spatial Channel Characterization for Urban Environments' System Analysis. Sensors 2020, 20, 5360.

Publisher's Note: MDPI stays neutral with regard to jurisdictional claims in published maps and institutional affiliations. 
(C) 2020 by the authors. Submitted for possible open access publication under the terms and conditions of the Creative Commons Attribution (CC BY) license (http://creativecommons.org/licenses/by/4.0/). 